\title{
Strategy in the Trial-production Phase of HIGGS Watch
}

\author{
Sun youxia ${ }^{1, a}$, Li jinliang ${ }^{2, b}$ \\ ${ }^{1}$ Key Laboratory of Mining Disaster Prevention and Control of Education Ministry, Shandong \\ University of Science and Technology, Qingdao, Shandong, 266590,China \\ ${ }^{2}$ College of Mechanical and Electronic Engineering, Shandong University of Science and \\ Technology, Qingdao, Shandong, 266590, China \\ alixuanci@163.com, bsdustlj|@163.com
}

Keywords: trial-production, process optimization, management control

Abstract. The paper discussed the problems in trial-production line for the HIGGS watch, which made against the success of putting the new product into mass production smoothly and introducing it to the consumer market on schedule. After analyzing the causes of the problems, a series of solutions such as communication with customers, process optimization, jig improvement, optimal allocation of machines and operators, introduction of automatic machine and means of management control were put forward.

\section{Introduction}

Before entering mass production, there is a trial-production phase. In the phase, product design can be perfected, processing flows optimized and Work standards developed.

Trial-production of new product is omitted or the phase doesn't receive due attention, such problems as poor manufacturability, low rate of finished products and high reworking rate will occur in mass production. As a result, the production will be interrupted frequently, expected capacity can't be reached and customers will complain about product quality and delayed delivery.

PEGATRON enterprise thinks highly of trial-production of new product. In order to avoid facing the problems stated above, a series of strategies are adopted in the trial-production phase of HIGGS intelligent watch, which is a new product and has a broad market prospect. Industrial Engineers (IEs) were responsible for the development and implementation of strategies and communicated with other specialized persons at any time for a problem.

\section{Design of Trial-production Line}

The flowchart, shop-floor layout, work instruction and the schedule must be prepared before design of trial-production line. Trial-production line of the watch was linear cellular layout, which consisted of four sections that were the board test section, assembly section, detection section and packaging section. Because each trial-production line is usually dedicated to a specific product family, the output in the trial-production stage is stable and small. Cellular layout is the best choice to realize efficiency and flexibility of trial-production. Constrained by the spatial structure of the trial production shop-floor, the production line adopted a linear layout, not $U$ layout with higher efficiency. The number and location of the workplace depended on the output of trial-production and characteristics of process.

\section{Analysis of Problem in Trial-production}

Problem Identification. After training the workers, the trial-production line of HIGGS watch began running. Two problems were particularly prominent in the trial-production phase of HIGGS watch. Firstly, some performance standards of watch were inconsistent with the expected demand of customers according to the results of quality inspection and the opinions of target customers. Lower productivity was another problem. The capacity should reach 600UPH easily by reference to current standard times and labor toll, but that's not the case. 
Cause Analysis. After investigation on the shop-floor, the causes of the problems stated above can be classified into five categories in figure 1. The figure illustrates only the most important factors, which interact with each other.

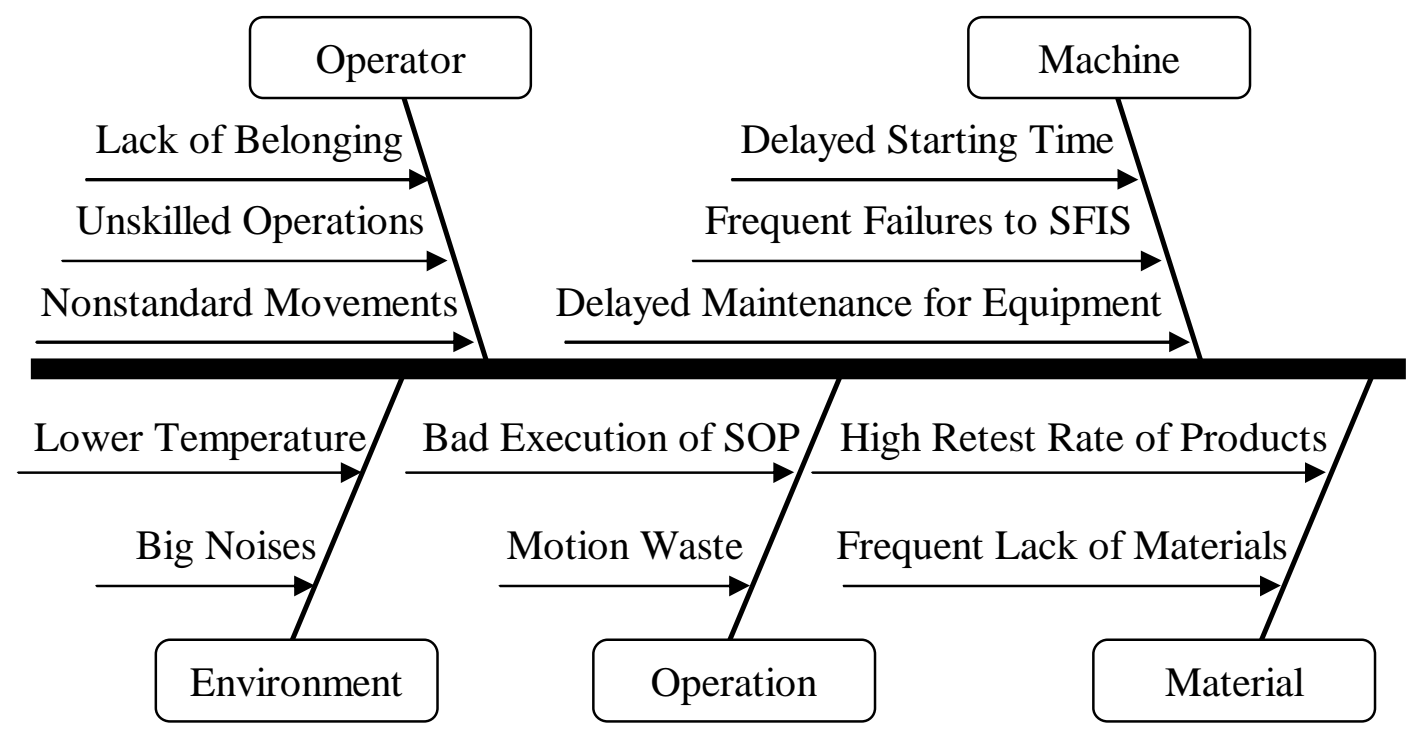

Fig 1 Causes for problems in trial-production

After a period of trial-production, it has been found that the starting-time was always delayed to 10AM, which was two hours later than the normal starting time. What's worse was the workers sometimes didn't start working at all. As a result, the effective working time was greatly reduced, and it's impossible to reach designed yield. The delay might come from the lax implementation of rules and regulations, also come from that the workers do not know what and how to do when some requirement for the watch from customers changed.

Shop-floor Integrated System (SFIS) essentially functioned as management information of trial-production, which played a very significant role in providing staff with data about material, machine, schedule and product quality etc in the trail-production line. Unfortunately, SFIS didn't work very well. The reason was that few people were be proficient in SFIS, and could not make it work again when the system was out of order. Consequently, the starting-time was delayed and production process was interrupted because of lack of information about material, machine, schedule and product quality etc. The product quality and the output must be affected.

In trial-production phase, the manufacturing technology and the production process for the HIGGS watch would change when the design of it changed. So Standard Operating Procedure (SOP) was not perfect. Of course, in such situation it is impossible to ask operators to perform a task in strict accordance with SOP. In addition, the relaxed management from the superior and the absence of belonging of workers could also make the operators be sneezed at the SOP.

Imperfect SOP made workers have no standards to follow when they faced some tasks, and they also ignored available SOP, both which would cause nonstandard movement and unskilled operation. Working together, nonstandard movement and unskilled operation resulted in motion waste and high retest rate of products. In the end, both the product quality and productivity have been reduced.

Work environment such as temperature and noises were age-old problem in many companies that was not easy to solve, but the enterprise has been trying to reduce their impact on workers. Materials flow couldn't be overlooked when the improvement of product quality and production was talked about. Congestion and lack of materials in some workplace came from frequent failures to SFIS and process load unbalance. Load unbalance could make workers at some process were too busy to rest for a moment, and labors at other station were more leisure. So materials were piled up in busy process, but in next process shortage of materials would occur. In addition, overwork of workers would cause defective products and low productivity. 


\section{Solution to Problem in Trial-production}

Three solutions to problems in trial-production for HIGGS watch were proposed as shown in table 1. The communication with customers first was underlined. The process optimization based on industrial engineering principle followed. At last, the means of management control were stated.

Table 1 Solutions to problems in trial-production for HIGGS watch

\begin{tabular}{|l|l|}
\hline \multicolumn{1}{|c|}{ Solutions } & Ways \\
\hline Communication with Customers & $\begin{array}{l}\text { inviting customers to test product performance in } \\
\text { production line; }\end{array}$ \\
\hline Process Optimization & $\begin{array}{l}\text { bottleneck optimization; } \\
\text { jig improvement; } \\
\text { optimal allocation of machines and operators; } \\
\text { introduction of automation; }\end{array}$ \\
\hline Management Control & $\begin{array}{l}\text { strengthening the daily management; } \\
\text { amending incentive mechanism; } \\
\text { developing a training plan; }\end{array}$ \\
\hline
\end{tabular}

Communication with Customers. In the trial-production phase of HIGGS watch, enterprises have invited the target customers to test product performance index in production line except quality inspection carried out by the enterprise itself, because the users know best about what they want. The performance standards of watch that were inconsistent with the expected demand of customers would be revised and amended timely. The corresponding production process and machining accuracy would also be adjusted. Consequently, the final design of products would be determined and SOP be perfected.

Process Optimization. Line balancing is the preferred method to improve process. It can keep the workload of the production in equilibrium, rationalize the operation process and reduce high retest rate of products, thus increasing yield and quality. The ways adopted in line balancing were as follows.

Such as the process of sticking foam, compacting battery, covering metal cover and attaching gasket to $\mathrm{MB}$, which produced less than other processes in an hour, were identified to be the bottleneck of production line for the HIGGS watch. Then motion analysis technology was used to split, assemble and adjust the critical movements of bottleneck process. It was a good idea that some tasks of bottleneck process were shared by other processes, or more labors were placed in the busy process. As a result, movements and processes would be standardized, and the line be balanced more.

Jig improvement is an effective way to improve line productivity. After repeated observation and practice, the jigs of covering metal cover and attaching gasket to MB could be integrated together, and a new jig was developed. The new jig not only hasn't affected the completion of the tasks, but also has reduced the number of movement and standard times. The production efficiency was improved.

In order to achieve the optimal allocation of machines and operators, the analysis of man-machine operation and spare capacity was carried out, and the improvement was followed. As a result, the utilization rate of machine and workers was improved greatly.

In the initial stage of trial-production, the task of locking the battery frame to the lower cover was done by a worker with an electric screwdriver. The simple equipment saved money but incurred the deviation of positioning and slipping of screw. It took operators a long time and a lot of attention to accomplish this task, which not only affected work efficiency but also caused high fatigue of workers. Considering the market potential of HIGGS watch, the company purchased full-automatic screw driving machine. Operation time was reduced greatly and the workers were freed from the tied and boring task.

Management Control. There were many ways of management control, but only three means here were mentioned. The enterprise strengthened the daily management of the shop-floor to ensure the 
staff start work on time and take each task seriously. The basic-level supervisors were asked to go to production line to guide, check, and supervise the activities of the subordinate.

Incentive mechanism was also amended, which tended to encourage staff to make constructive proposal that could improve the properties of product and processing technology.

Otherwise, a training plan was developed, which mainly included three modules. The first module severed to inform the staff of the importance of trial-production. This was considered important content, which could increase the sense of belonging of employees, then improve the enthusiasm and positivity of workers. The second module was used to give all trainees the meaning, content, usage and maintenance of the SFIS. With the knowledge of the SFIS, the problems such as material confusion and line interruption etc caused by the system failure could be eliminated. Due to the continuous improvement of the product itself, processing technology was constantly changing, so the technical skills of workers should be updated. The third module was for skills training of employees, which would promote the standard movement and skilled operation of workers.

\section{Conclusions}

It has become a competition focus among enterprises that launching products that meet consumer needs in the shortest possible time. In order to succeed in the stiff competition, companies should not overlook the trial-production. In the trial-production phase, the performance parameters of HIGGS watches that satisfy the market were perfected, machining technology and the process flow were improved, and other problems such as the laxity of workers were identified and solved. All of these could guarantee that the HIGGS watches were put into mass production smoothly and introduced to the consumer market on schedule.

\section{References}

[1] Xia xuhui: submitted to Journal of Computer Integrated Manufacturing Systems(2017).

[2] Li dongwen, Lu zhiqiang: submitted to Journal of Machinery(2017).

[3] Li aiping, Lu li : submitted to Journal of Tongji University(2017).

[4] He fuwei: submitted to Journal of Yunnan Chemical Technology(2017).

[5] M.A. Bao zikun: Study on Pre-production Preparation of CH Company (2014). 